\title{
Reversible Data Hiding Using Pixel Order Exchange
}

\author{
Hsiao-Ling Wu, Chin-Chen Chang, and Thai-Son Nguyen
}

\begin{abstract}
Reversible data hiding is a technique that extracts secret data exactly and recovers the cover image completely. Many reversible data hiding schemes have been proposed in the literature for various goals. However, many drawbacks are discovered in these schemes, which are low visual quality, limited embedding capacity and a large size of the location map. In this paper, we propose a new reversible data hiding scheme using pixel order exchange to enhance the embedding capacity and the visual quality of the stego image. In addition, the size of the location map in our proposed scheme is very small. In the proposed scheme, the cover image is processed first. Then, the order of pixels in a pair is exchanged to embed secret data, while guaranteeing only a small distortion of the stego image. Our experimental results indicated that the proposed scheme provides larger embedding capacity and better image quality than some previous schemes.
\end{abstract}

Index Terms-Reversible data hiding, pixel order exchange, good image quality, high embedding capacity.

\section{INTRODUCTION}

Reversible data hiding is a technique that extracts the secret data exactly and recovers the original cover image completely after the secret data have been completely extracted. Many reversible data hiding schemes have been proposed in the literature for various goals. Most reversible data hiding schemes [1]-[15] can be classified into two types, i.e., difference expansion-based (DE-based) schemes [1]-[7] and histogram-based schemes [8]-[15]. In DE-based schemes, the main idea is to use the difference value of pixels for embedding data instead of using the original values of the pixels. In 2003, Tian [1] first introduced a DE-based data hiding scheme that provided high embedding capacity (EC) and ensured low distortion. However, a large location map was required in this scheme to obtain reversibility. Then, Tian's scheme was developed by Hu et al. [2] to reduce the size of the location map. Hu et al.'s scheme determined the directions in which predicted errors shifted according to the embedding capacity. Then, they used the corresponding formula to embed secret data into the cover image. Recently, the DE-based algorithm has been researched extensively and developed in various fields [2]-[7].

Histogram-based schemes were first introduced by $\mathrm{Ni}$ et al. [8], and they are another important type of reversible data hiding. In Ni et al.'s scheme, the peak point of the image histogram was determined and used to embed the secret data.

Manuscript received October 10, 2016; revised March 30, 2017.

H. L. Wu, C. C. Chang, and T. S. Nguyen are with the Department of Information Engineering and Computer Science, Feng Chia University, Taichung, Taiwan (e-mail: wuhsiaoling590@gmail.com, alan3c@gmail.com, thaison@tvu.edu.vn).

T. S. Nguyen is with the Department of Information Technology, Tra Vinh University, Tra Vinh Province, Vietnam.
By doing so, each pixel in the cover image was modified by one value, at most. As a result, their scheme provided a stego image with high visual quality, but the scheme had limited embedding capacity. To increase the embedding capacity and the visual quality of the stego image, Luo et al. [13] selected the median pixel of each block to structure a reference sub-image. However, the average embedding capacity of their scheme was quite a bit lower than $0.1 \mathrm{bpp}$ for a single embedding level, because the reference pixels or reference sub-images were not used for embedding data. Many reversible, histogram-based data hiding schemes [9]-[12] have been proposed to further improve the quality of the stego images and the embedding capacity provided by $\mathrm{Ni} e t$ al.'s scheme.

In this paper, we presented a new type of scheme for reversible data hiding. Different from the DE-based and histogram-based schemes, the proposed scheme was designed based on exchanging the order of the pixels in a pair to embed secret data. In the proposed scheme, half of the pixels, at most, in the cover image are altered by one value. Therefore, the proposed scheme can guarantee good visual quality stego images while embedding a large amount of secret data. In addition, the location map generated during the embedding process is encoded and embedded into the cover image directly to ensure that no extra information is required on the receiver side of the proposed scheme.

\section{PROPOSED SCHEME}

In this section, we propose a new reversible data hiding scheme for digital images. The proposed scheme is divided into two phases, i.e., the embedding phase and the extracting phase. These phases are discussed in Subsections II.A and II.B, respectively.

\section{A. Embedding Phase}

In this subsection, we describe the details of the embedding phase of the proposed scheme. Fig. 1 shows all of the main steps of the embedding phase.

Initially, a cover image I made up of $M \times N$ pixels is divided into two regions, i.e. an embeddable region and an unembeddable region. The embeddable region is partitioned into pairs of pixels to carry the secret data, and the least significant (LSB) bits of the pixels are in the unembeddable region. The unembeddable region is used to contain the location map that is generated during the embedding process. The details of the algorithm used in the embedding phase are as follows:

Step 1 (Image division): The cover image $I$ is divided into two regions, i.e., the embeddable region and the un-embeddable region. The un-embeddable region consists of two lowest rows of the cover image. The LSBs of pixels in 
the unembeddable region are emptied and used to record information concerning the location map LM. Therefore, the LSBs of these pixels in the unembeddable region must be extracted and concatenated into secret data $B$ to generate the embedded data $S$. The pixels in the embeddable region are partitioned into pairs of pixels, (, ), where ,, and $M$ and $N$ are the height and width of the cover image, respectively.

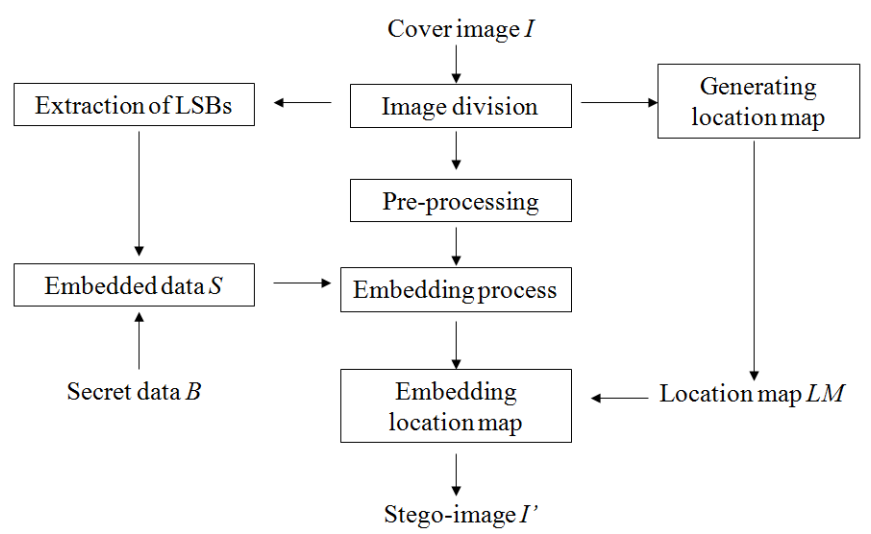

Fig. 1. Flowchart of the embedding phase.

Step 2 (Generating the Location map): To avoid the overflow/underflow problem, for the pair of pixels, (,), if , the corresponding location $L M((j+1) / 2)$ of this pair in the location map $L M$ is set to 1 , and the value of is modified to . Otherwise, $\operatorname{LM}((j+1) / 2)=0$. Note that, there are only a few exception pairs that may causes overflow/underflow, meaning that there are only a few values of " 1 " in the location map. In addition, a lossless compression algorithm, i.e., JBIG-kit [16], is used to reduce the size of the location map.

Step 3 (Pre-processing): For each pair of pixels, (,), the second pixel is set as, and the difference value is computed as. Then, the histogram of these difference values is constructed, where and.

Step 4 (Embedding process): Determine the positive peak point $P K$ of the histogram. Then, embed the data $S$ into the cover image:

- For the pair of pixels, (,):

- If, is shifted to 1 , meaning that .

- If , embed a bit $b \in\{0,1\}$ by exchanging the order of the two pixels of the pair using Equation (1):

$$
\left(s p_{i, j}, s p_{i, j+1}\right)= \begin{cases}\left(p_{i, j}, p_{i, j+1}\right) & \text { if } b=0, \\ \left(p_{i, j+1}, p_{i, j}\right) & \text { if } b=1 .\end{cases}
$$

- Otherwise, the pair of pixels, (,), are not altered.

This step is terminated if all bits of $S$ are embedded. Fig. 2 shows the value of $d_{k}$ during the embedding process.

Step 5 (Embedding the location map): Since the LSBs of the pixels in the unembeddable region are recorded in Step 1, replace these LSBs by the compressed location map generated in Step 2 to generate the stego image.

\section{B. Extracting Phase}

When the stego image and the peak value $P K$ are available, the receiver performs the extracting algorithm to extract the secret data and reconstruct the original cover image. Details of the corresponding data extracting algorithm are provided below.
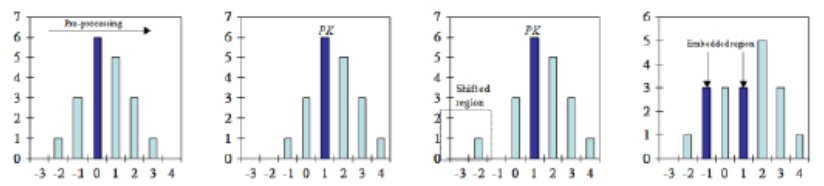

(a) Original (b) After pre-processing (c) After shifting (d) After embedding

Fig. 2. Illustration of the value of $\mathrm{dk}$ in the embedding phase.

Step 1 (Extraction of the location map): Extract the LSBs of the pixels in the unembeddable region to obtain the compressed location map. Then, decompress them to reconstruct the original location map $L M$.

Step 2 (Data extraction): Scan each pair of pixels (,) in the embeddable region of the stego image, where and.

For the pair of pixels, (,), calculate.

- If, is shifted to 1 , meaning that.

- If, a secret bit $b$ is extracted from the pair. The secret bit $b$ is calculated by Equation (2):

$$
b= \begin{cases}0 & \text { if } d_{k}=P K \\ 1 & \text { if } d_{k}=-P K .\end{cases}
$$

Step 3 (Image recovery): The second pixel of each pair of pixels is modified to . The original pixels in the embeddable region are reconstructed according to the location map $L M$. If the corresponding location map value $L M((j+1) / 2)$ is " 1 ," the pair of pixels in the embeddable region is recovered as $($,$) .$ Otherwise, the pair of pixels remains unchanged. Finally, to obtain the original cover image $I$, the LSBs of the pixels in the unembeddable region are recovered from the embedded data $S$ by substituting the LSBs.

\section{EXPERIMENTAL RESULTS}

In order to demonstrate the performance of our proposed scheme, four common test images were used as the cover images, i.e., "Lena," "Airplane," "Peppers," and "Tiffany." These images are shown in Fig. 3. Secret data $B$ comprised a random bit stream, and the bit stream was generated by a pseudo random generator. In our experiments, MATLAB R2010a was used to program the proposed scheme and the other four previous schemes.

In the proposed scheme, only one pixel in each pair of pixels will be modified by one value during the data embedding process, and the other pixels remain unchanged. Therefore, the PSNR of the stego image compared with the original version is at least:

$$
10 \log _{10}\left(\frac{255^{2}}{1 / n}\right)=10 \log _{10}\left(2 \times 255^{2}\right)>51 \mathrm{~dB},
$$

where $n$ is the number of pixels in each pair.

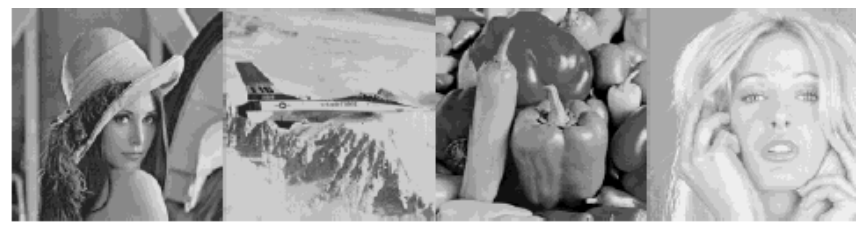

Fig. 3. Test images: (a) Lena; (b) Airplane; (c) Peppers; (d) Tiffany.

Fig. 4 shows four stego images after fully embedding 
capacity and their corresponding PSNRs. Obviously, the average value of PSNR is greater than $52 \mathrm{~dB}$, meaning that the stego image is imperceptible by the human eye.

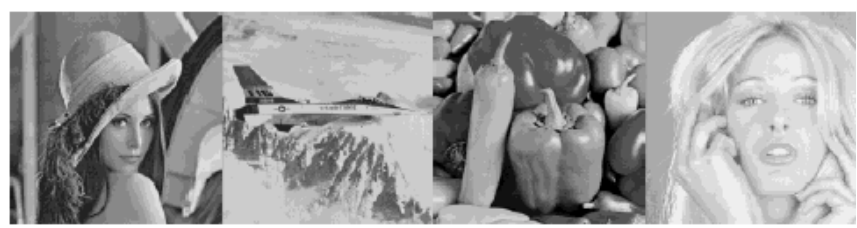

Fig. 4. Stego images after fully embedding capacity.

Fig. 5 compares the performances of the proposed schemes and three previous well-known schemes, i.e., the schemes of Tian [1], Ni et al. [8], and Luo et al. [13]. In this experiment, we varied capacity from 1,000 bits to 15,000 bits with a step size of 1,000 bits. The top curves in Fig. 5 show that the proposed scheme and the scheme in [13] had similar behavior In some cases, the results of the scheme in [13] were slightly better than that of the proposed scheme. However, it is clear that the scheme in [13] is only useful in cases in which low embedding capacity is required. When it is necessary to embed larger amounts of secret data, the proposed scheme achieves better quality of the stego images than three previous schemes [1], [8], [13].

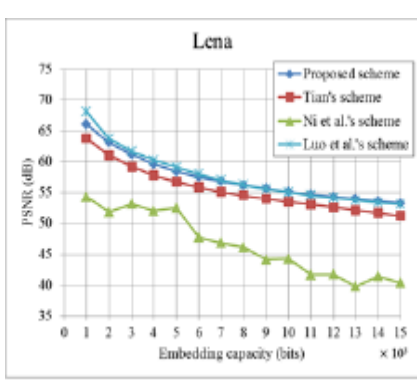

(a)

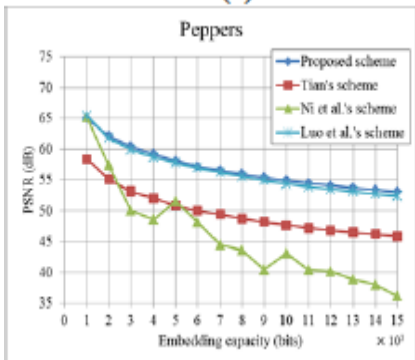

Fig. 5. Comparison of the performances of four schemes.

TABLE I: COMPARISON OF PSNR (IN DB) BETWEEN THE PROPOSED SCHEME AND FOUR PREVIOUS SCHEMES [1], [2], [8], [13] FOR AN EC OF 10,000 BITS

\begin{tabular}{lccccc}
\hline \hline $\begin{array}{c}\text { Schemes } \\
\text { Images }\end{array}$ & $\begin{array}{c}\text { Ni } \text { et al. } \\
{[8]}\end{array}$ & Tian [1] & $\begin{array}{c}\text { Hu et al. } \\
{[2]}\end{array}$ & $\begin{array}{c}\text { Luo et al. } \\
{[13]}\end{array}$ & Proposed \\
\hline Lena & 44.19 & 53.46 & 56.46 & 55.05 & 56.75 \\
Airplane & 51.70 & 48.09 & 57.24 & 55.75 & 56.82 \\
Peppers & 43.11 & 47.69 & 53.36 & 54.38 & 55.71 \\
Tiffany & 46.68 & 48.90 & 53.57 & 54.43 & 55.62 \\
Average & 46.42 & 49.54 & 55.16 & 54.90 & 56.23 \\
\hline \hline
\end{tabular}

Table I compares the PSNR (in $\mathrm{dB}$ ) of the proposed scheme and four previous schemes [1], [2], [8], [13] for an EC of 10,000 bits. Table 1 shows that the proposed scheme, on average, outperformed $\mathrm{Hu}$ et al.'s scheme, Luo et al.'s scheme, Tian's scheme, and Ni et al.'s scheme by 1.07, 1.33, 6.69 , and $9.81 \mathrm{~dB}$ for an embedding capacity of 10,000 bits. The proposed scheme obtained better PSNRs than the four previous schemes because only the second pixel is modified by one value for each pair of pixels, and the first pixel remains unchanged. This process means that only half of the pixels in the image are modified. In contrast, in four other previous schemes [1], [2], [8], [13], all of the pixels of the image are modified for embedding the secret data. Thus, the image is significantly distorted after the secret data are embedded.

\section{CONCLUSIONS}

In this paper, we presented a new scheme for reversible data hiding. In the proposed scheme, the order of the pixels in the pair is exchanged to embed secret data. Therefore, only one pixel in the pair is modified by one value during the embedding process, which improves the visual quality of the stego image. The proposed scheme can provide better visual quality after embedding the same EC than the previous schemes. In the future, we will focus on research to further improve both the visual quality of the stego image and EC.

\section{REFERENCES}

[1] J. Tian, "Reversible data embedding using a difference expansion," IEEE Trans. Circuits Syst. Video Technol., vol. 13, no. 8, pp. 890-896, Aug. 2003.

[2] Y. Hu, H. K. Lee, and J. Li, "DE-based reversible data hiding with improved overflow location map," IEEE Trans. Circuits Syst. Video Technol., vol. 19, no. 2, pp. 250-260, Feb. 2009.

[3] W. L. Tai, C. M. Yeh, and C. C. Chang, "Reversible data hiding based on histogram modification of pixel differences," IEEE Trans. Circuits Syst. Video Technol., vol. 19, no. 6, pp. 906-910, Jun. 2009.

[4] L. Luo, Z. Chen, M. Chen, X. Zeng, and Z. Xiong, "Reversible image watermarking using interpolation technique," IEEE Trans. Inf. Forensics Security, vol. 5, no. 1, pp. 187-193, Mar. 2011.

[5] C. C. Chang, T. S. Nguyen, and C. C. Lin, "A reversible data hiding scheme for VQ indices based on absolute difference trees," KSII Trans. on Internet Inf. Syst., vol. 8, no. 7, pp. 2572-2589, Jul. 2014.

[6] X. Wang, X. Li, B. Yang, and Z. Guo, "Efficient generalized integer transform for reversible watermarking," IEEE Signal Process. Lett., vol. 17 , no. 6 , pp. $567-570$, Jun. 2010.

[7] F. Peng, X. Li, and B. Yang, "Adaptive reversible data hiding scheme based on integer transform," Signal Process, vol. 92, no. 1, pp. 54-62, Jun. 2012.

[8] Z. Ni, Y. Q. Shi, N. Ansari, and W. Su, "Reversible data hiding," IEEE Trans. Circuits Syst. Video Technol., vol. 16, no. 3, pp. 354-362, Mar. 2006.

[9] X. T. Wang, C. C. Chang, T. S. Nguyen, and M. C. Li, "Reversible data hiding for high quality images exploiting interpolation and direction order mechanism," Digit. Signal Process, vol. 23, no. 2, pp. 569-577, Mar. 2013.

[10] H. T. Wu, J. L. Dugelay, and Y. Q. Shi, "Reversible image data hiding with contrast enhancement," IEEE Signal Process. Lett., vol. 22, no. 1 , pp.81-85, Jan. 2015.

[11] X. L. Li, W. M. Zhang, X. L. Gui, and B. Yang, "A novel reversible data hiding scheme based on two-dimensional difference histogram modification," IEEE Trans. Inf. Forensics Security, vol. 8, no. 7, pp. 1091-1100, Jul. 2013.

[12] X. L. Li, B. Li, B. Yang, and T. Y. Zeng, "General framework to histogram-shifting-based reversible data hiding," IEEE Trans. Image Process., vol. 22, no. 6, pp 2181-2191, Jun. 2013.

[13] H. Luo, F. X. Yu, H. Chen, Z. L. Huang, H. Li, and P. H. Wang, "Reversible data hiding based on block median preservation," Inf. Sci., vol. 181, no. 2, pp. 308-328, Jun. 2011.

[14] T. S. Nguyen, C. C. Chang, and M. C. Lin, "Adaptive lossless data-hiding and compression scheme for SMVQ indices using SOC," The Smart Comput. Rev., vol. 4, no. 3, pp. 230-245, Jun. 2014.

[15] C. C. Chang, H. L. Wu, and T. F. Chung, "Applying Histogram Modification to Embed Secret Message in AMBTC," in Proc. Int. Conf. on Intell. Inf. Hiding and Multimedia Signal Process, 2014, pp. 489-492.

[16] M. Kuhn. JBIG-KIT. [Online]. Available: http://www.cl.cam.ac.uk/ mgk25/jbigkit 


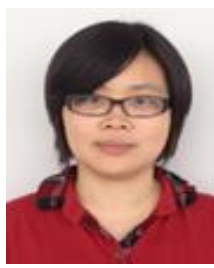

Hsiao-Ling Wu received the BS degree in the Department of Applied Mathematics from Feng Chia University, Taichung, Taiwan in 2007. She is currently pursuing her Ph.D. degree in information engineering and computer science from Feng Chia University, Taichung, Taiwan. Her current research interests include electronic commerce, information security, image processing, cryptography, and mobile

communications.

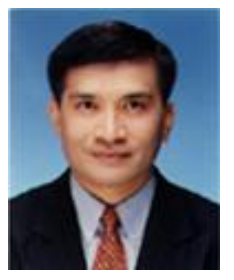

Chin-Chen Chang received his BS degree in applied mathematics in 1977 and the MS degree in computer and decision sciences in 1979, both from the National Tsing Hua University, Hsinchu, Taiwan. He received his $\mathrm{Ph} . \mathrm{D}$ in computer engineering in 1982 from the National Chiao Tung University, Hsinchu, Taiwan. Since February 2005, he has been a chair professor of Feng Chia University. In addition, he has served as a consultant to several research institutes and government departments. His current research interests include database design, computer cryptography, image compression and data structures.

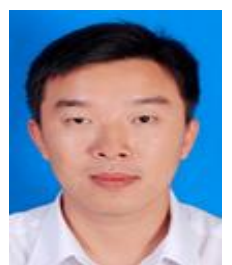

Thai-Son Nguyen received the bachelor degree in information technology from Open University, HCM city, Vietnam, in 2005. From December 2006, he has been lecturer of TraVinh University, TraVinh, Vietnam. In 2011, he received M.S. degree in computer sciences from FengChia University, TaiChung, Taiwan. He is currently pursuing the Ph.D. degree with the Department of Information Engineering and Computer Science, Feng Chia University, Taichung, Taiwan. His current research interests include data hiding, image processing, and information security. 